\title{
Using of Artificial Neural Network and COMSOL Multiphysics for Modeling of Pervaporation Process \\ Mansoor Kazemimoghadam ${ }^{1 *}$ and Zahra Amiri ${ }^{2}$ \\ ${ }^{1}$ Department of Chemical Engineering, Malek-Ashtar University of Technology, Tehran, Iran. \\ ${ }^{2}$ Department of Chemical Engineering, South Tehran Branch, Islamic Azad University, Tehran, Iran.
}

\begin{abstract}
The researcher in this study used a Feed Forward multilayer perceptron neural network with a back propagation algorithm and Levenberg-Marquardt function with two inputs and two outputs. The Tansig transfer function was used for the hidden layer and Purelin was used for the output layer; five neurons were defined for the hidden layer. After data processing, 70 percent of the data was allocated for learning, 15 percent was allocated for validation, and 25 percent was allocated for testing. The output values of Artificial Neural Network modelling were compared with the real values of pervaporation for separation of water from Ethanol, Acetone, and Butanol. The results revealed that the proposed model had a good performance. Moreover, the output of COMSOL software for pervaporation of five different alcohols was compared with the real values, and the error percentage of the actual amount of flux was calculated with the modeling value by means of related membranes. The results of COMSOL modeling showed that the error percentages of 3.049, 3.7, 3.51, 2.88, and 3.82 were respectively achieved for dehydration process of Acetone, Butanol, Ethanol, Isopropanol and Methanol.
\end{abstract}

KEYWORDS: Modeling, Dehydration, Polymer membrane, COMSOL Multiphysics, Artificial Neural Network

(C) 2017 mahendrapublications.com, All rights reserved

\section{INTRODUCTION}

Use of pervaporation for separation of organic compounds has attracted the attention of many researchers in recent years. Pervaporation is one of the complicated membrane separation processes in which transfer through non-porous (non)polymer takes place in three phases of absorption, penetration, and evaporation (or disposal) [1-5]. As a result, selectivity and permeation is generally based on the interaction between membrane and penetrating molecules, the size of leaking molecules, and the empty volume of membrane. This process is generally very good for excluding little impurity from a liquid mixture. In general, pervaporation is accompanied with penetrating material phase change from liquid to gas. The passed product through the membrane will be separated as low-pressure steam from the other side of the membrane. The product will be collected after changing into liquid. In fact, this process is the known evaporation process, while a membrane is used between the two phases of liquid and gas. The presence of membrane adds selectivity to the process and increases the advantages of the process. With the help of such process, it is possible to separate two liquids from each other [6]. Due to such advantages as excellent performance and high energy efficiency, this process has recently gained attention of many industries. In most pervaporation processes, the driving force is the pressure difference between the feed stream and the permeate stream. The vacuum pump provides the driving force for mass transfer of components [7].

The results of this study by use of ANN reflected a suitable accuracy. The graph of error percentage for the real outputs of separation factor and flux and the modeled separation factor and flux by the related membranes for pervaporation performance were drawn in dehydration of ethanol, acetone, and butanol. Moreover, the error percentage for the real flux and modeled flux by the related membranes for each of the five alcohols were modeled by the COMSOL Multiphysics.

\section{MATERIALS AND METHODS \\ 2.1. Artificial Neural Network (ANN)}

Recently, there has been a number of research conducted on data processing for problems for which there is no solution, or problems that are not easily solvable. The ANN pattern is inspired by the neural system of living organisms that includes some constituent units called 'Neuron'. Most of the neurons are composed of the three main parts including cell body (that includes necluous and other protective parts), dendrites, and axon. The last two parts are the communicative parts of the neuron. Figure 1 displays the structure of a neuron.

Dendrites, as electric signal receiving areas, are composed of cell fibers with unsmooth surface and many splitted extensions. That is why they are called tree-like receiving networks. The dendrites transfer the electrical signals into cell nucleous. The cell body provides the required energy for neuron activity that can be easily modelled through an addition and comparison with threshold level. Unlike Dendrites, axon has a smoother surface and fewer extensions. Axon is longer and transfers the received electro-chemical signal from the cell nucluous to other neurons. 


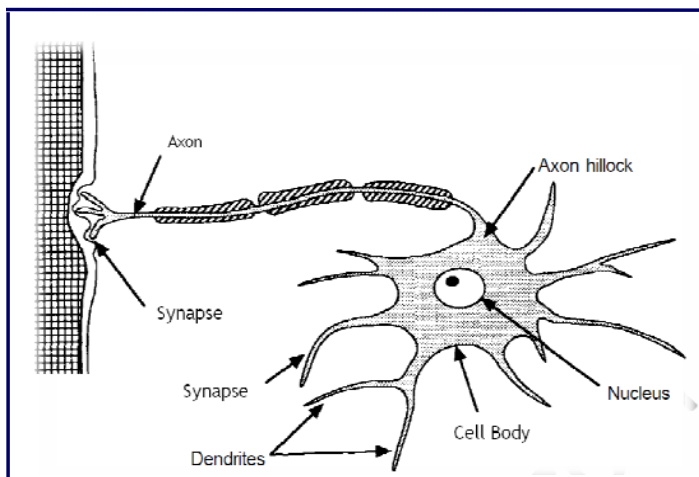

Figure.1 Major parts of a biological cell

The confluence of a cell's axon and dendrites is called synapse. Synapses are small functional structural units that enable the communication among neurons. Synapses have different types, from which one of the most important ones is the chemical synapse.

Artificial neural cell is a mathematical equation in which $p$ represents an input signal. After strengthening or weakening as much as a parameter $\mathrm{w}$ (in mathematical terms, it is called weight parameter), an electric signal with a value of pw will enter the neuron. In order to simplify the mathematical equation, it is assumed that the input signal is added to another signal with $b$ value in the nucluous. Before getting out of the cell, the final signal with a value of pw+b will undergo another process that is called "Transfer function" in technical terms. This operation is displayed as a box in Figure 2 on which $\mathrm{f}$ is written. The input of this box is the pw+b signal and the output is displayed by $a$. Mathematically, we will have:

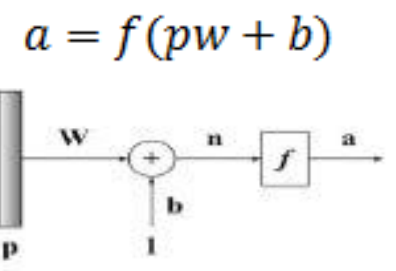

Figure 2 Mathematical model of a neuron

Putting together a great number of the above-mentioned cells brings about a big neural network. As a result, the network developer must assign values for a huge number of $\mathrm{w}$ and $\mathrm{b}$ parameters; this process is called learning process.

Within the structure of neural networks, sometimes it is needed to stack up a number of neurons in a layer. Moreover, it is possible to take advantage of neuron crowds in different layers to increase the system efficiency. In this situation, the network will be designed with a certain number of inputs and outputs too; while the difference is that there would be more than one layer (instead of having only one layer). In this manner (multi-layer network), the input layer is the layer through which the inputs are given to the system, the output layer is the layer in which the desired the results are delivered, and the other layers are called hidden layer. Figure 3 displays a neural network with three layers. Input layer, output layer, and hidden layer (that is only one layer in this figure). Through changing the number of hidden layers, and changing the number of present neurons in each layer, it is possible to enhance the network capabilities [8].

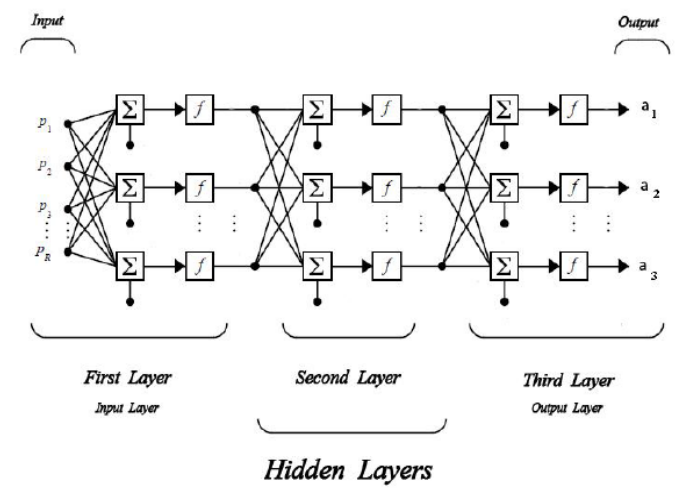

Figure. 3 A schematic view of Neural Network and its constituent layers

\subsection{COMSOL Multiphysics software}

COMSOL Multiphysics is modeling software that is able to go through the whole phases in modeling process. This modeling software is able to go through each and every phase of modeling as below:

- $\quad$ Drawing the geometrical structure of the model;

- $\quad$ Model meshing;

- $\quad$ Displaying model's principal physique;

- $\quad$ Model solvation; and

- $\quad$ Displaying the results of modeling in a graphical manner.

Due to existence of default physical structures in this software, model creation can be done very quickly. By use of this software, it is possible to analyze a wide range of mechanical and electro-magnetic structures. It is possible to define the materials' characteristics, resource parts, and the approximate borders of objects as arbitrary functions of independent variables in this software.

\subsection{Modeling dehydration of organic compounds by use of Neural Network}

In this research, the influence of ANN input parameters (volumetric flow and temperature) as well as the feed characteristics (the feeds are the network output) (separation factor and flux) on the efficiency of dehydration process. Two ANNs were designed for analysis of the separation factor and flux parameters. Feed-forward multilayer perceptron ANN and Levenberg-Marquardt function with two inputs and two outputs were used. The Tansig transfer function was used for the hidden layer, and Purelin was utilized for the output layer. Five neurons were determined for the hidden layer. After data processing, 70 percent was dedicated for learning, 15 percent was dedicated for validation, and the remaining 15 percent was dedicated for testing. Such organic compounds as ethanol, acetone, and butanol were selected in this research; and, Matlab version R2012a (7.14.0.739) was used. The results

\section{Mansoor Kazemimoghadam \& Zahra Amiri}


of the study are displayed in figure 4 . Figure 4 displays a schematic view of a two-layer ANN with only one hidden and output layer. The inputs are multiplied by a w value, and there is a bias factor (b) that is added to the input (bias is a fixed value that is added to the input in order to increase the accuracy). Afterward, the result will undergo a function and the resulted value will be multiplied by a weight and added with a bias. The final result will pass another function (with different form and functionality) and output is made. There are five neurons and two inputs on the first layer; however, the number of neurons in the output layer is the same as the number of outputs.

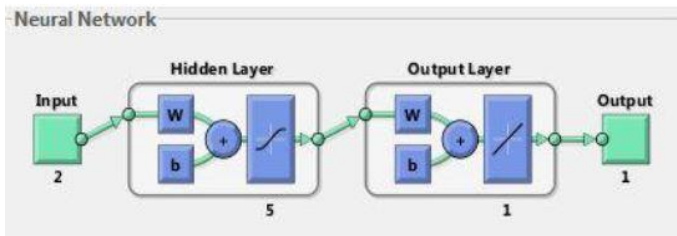

Figure. 4 A schematic view of the ANN

The following points about the algorithms must be considered:

The Data Division compartment totally scrambles the defined data for the system. This compartment randomly defines the Train, Validation, and Test data, so that there will be samples from everywhere of the environment.

Levenberg-Marquardt function was used in Training phase.

The Mean Squared Error (MSE) functions for performance measurement.

The default settings were used for derivative issue.

The number of data for modeling ethanol dehydration was 326. By use of polydimethylsiloxane and Polyvinylidene fluoride membrane for the output of separation factor, the following results were achieved [9]:

The whole procedure is displayed through some status bars in the progress compartment. The initial values are displayed on the left side of the status bar, and the present value is displayed on the right side.

Epoch is accepted from iteration 0 to 1000 . It means the weights consecutively changed for 1000 times based on the Levenberg-Marquardt function, and the training procedure was done. If the iteration number reaches 1000 , the procedure stops (here it stopped at 24). There was no limit for time (but it could be set for training to stop after 30 seconds for example).

The performance rate was 3.08 at the beginning. If this rate reaches 0 , the error would be acceptable. Finally, the error rate reached 0.00403 .

Gradient is the error function and it means the value of derivatives. The range for this variable starts from 9.77, and would be acceptable if it reaches $1.00 \mathrm{e}-05$. Finally, it reached 0.00171 .

$\mathrm{Mu}$ is one of the Levenberg-Marquardt algorithm parameters.

Validation check is the maximum number of times that network failure can be tolerated.

The performance graph shows the number of phases based on the errors. As shown in Figure 5, the network performance for Train, Validation, and Test has decreased to an acceptable level. Phase 18 that is marked with a circle was the best validation performance; i.e. there were fewer errors before the circle, and excessive training phase started after the circle.

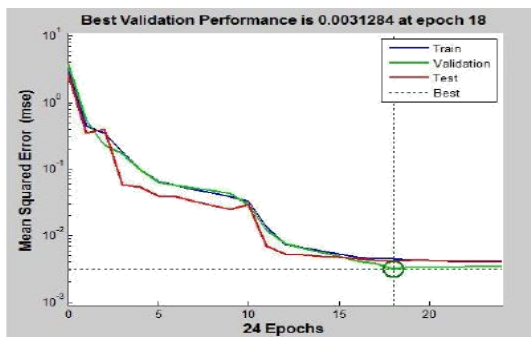

Figure. 5 The water- ethanol dehydration performance by Polydimethylsiloxane polymer membrane and Polyvinylidene fluoride

The training state graph shows different status in training phase. The first graph is for gradient error function. The second is for $\mathrm{Mu}$, and the third is for validation fail. Regarding the fact that the third graph reached 6 in the vertical axis and stopped, it shows failure. Moreover, the validation fail graph shows that the system has been stable for 18 times, and failed 6 times afterward; consequently, excessive training happened for it.

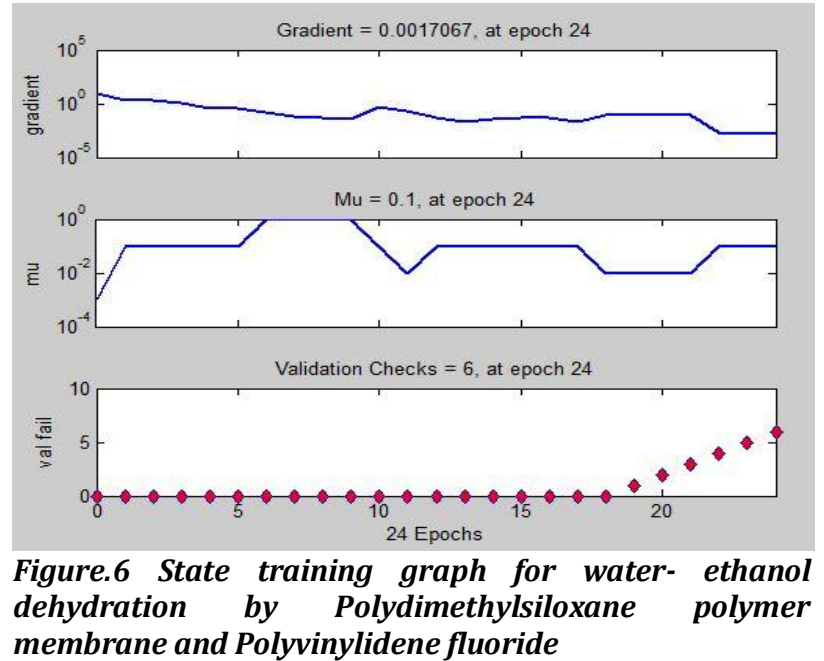

The regression graph demonstrates regression separately. The horizontal axis displays the outputs of the target parameters (in fact, what to be achieved). The vertical axis displays the ANN output. As a result, the graph is drawn based on these two parameters. If the ANN would be able to model exactly, the graph should be placed the $Y=T$ line (a line with a slope of 1 that passes the origin of coordinates). In order to statistically calculate the best line with the lowest error, the linear equation achieved in all graph must be used:

$$
\text { Output }=0.99 \times \text { Target }+0.13
$$

Mansoor Kazemimoghadam \& Zahra Amiri 
The result would be better if the value of $F$ will be closer to 1. This shows the fitting desirability, i.e. there is a low difference between the target outputs and the ANN outputs in the modeling. In general, the regression coefficient for all the data was calculated to be 0.99871 that is considered a very good result.
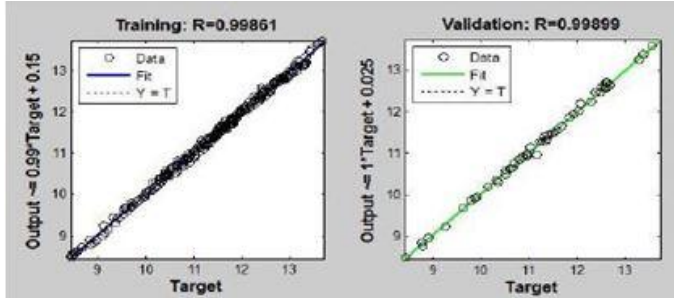

Test: $\mathbf{R}=0.99898$
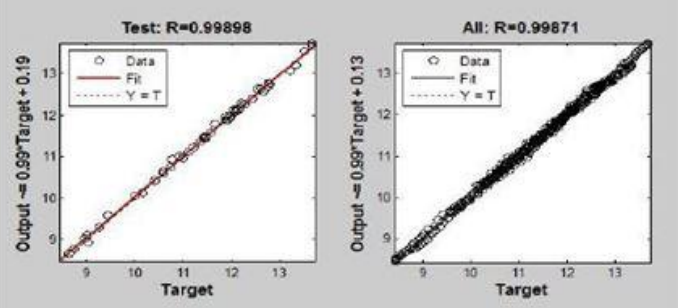

Figure.7 Regression graph for water-ethanol dehydration by Polydimethylsiloxane polymer membrane and Polyvinylidene fluoride

Figure 8 displays the water- ethanol figure graph for Polydimethylsiloxane polymer membrane and Polyvinylidene fluoride. According to this figure, with increase of temperature and decrease of volumetric flow rate in ethanol dehydration, the separation factor increases in the beginning, and decreases afterward.

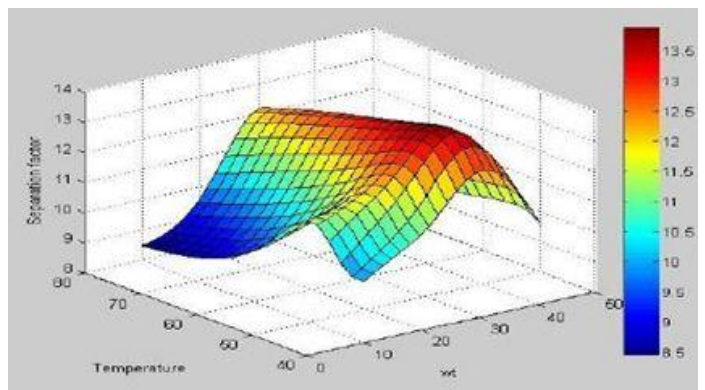

Figure. 8 Figure graph for Water- ethanol dehydration by Polydimethylsiloxane polymer membrane and Polyvinylidene fluoride

The graph for calculation of error percentage of real output and modeling output is as figure 9. Using the related formula, the error percentage of the real data and modeling data can be achieved. The lower percentage of error would be more desirable. As an example, a number of accidental cases of data were selected, and their error percentage was calculated. A comparison between the separation factor of the real values and the modeling values was conducted then. The results of the comparison revealed that there was a little difference between the real data and the modeling data. As a result, the modeling has been successful.

As shown in Figure 9, with increase of temperature and decrease of volumetric flow rate, the separation factor increases first, and decreases afterward. The cause of this phenomenon is that the propulsion increases with the increase of temperature at first. However, as the temperature continuously raises the difference between the water- ethanol solubility and diffusion rate decreases, and the separation factor declines accordingly.

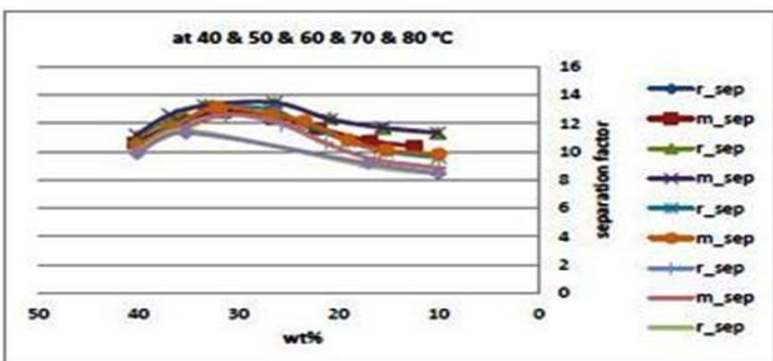

Figure. 9 A comparison between the error percentage of real separation factor and the modeling separation factor for water and ethanol Polydimethylsiloxane polymer membrane and Polyvinylidene fluoride

The output of overall flux in water- ethanol dehydration through ethanol Polydimethylsiloxane polymer membrane and Polyvinylidene fluoride, with 326 data is as follows. As shown in Fig. 10, in the performance graph, the best validation performance was in the twenty-first repetition, and the excessive learning started afterward.

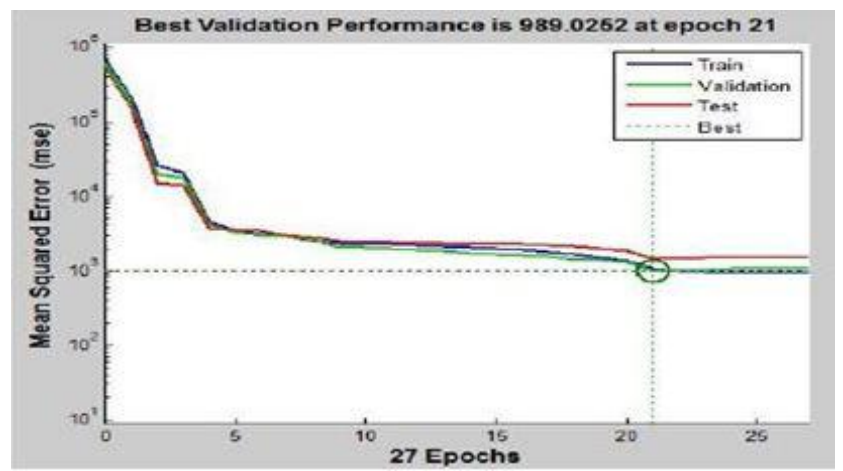

Figure.10 Performance graph for water- ethanol dehydration by ethanol Polydimethylsiloxane polymer membrane and Polyvinylidene fluoride

Figure 11 displays the regression graph. As depicted in ALL graph, the best line with the lowest error would be as follows:

Output $=0.99 \times$ Target +92

\section{Mansoor Kazemimoghadam \& Zahra Amiri}



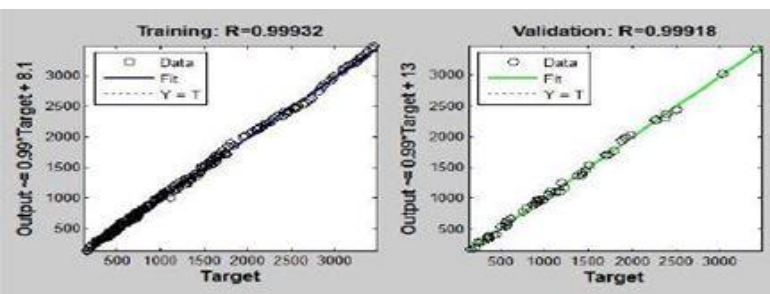

Test: $\mathrm{R}=0.99868$
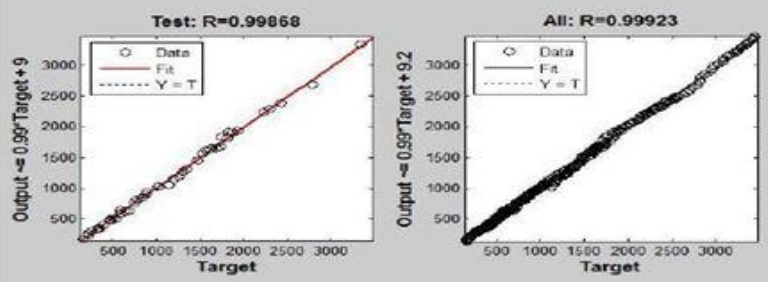

Figure.11 Regression graph for water- ethanol dehydration by ethanol Polydimethylsiloxane polymer membrane and Polyvinylidene fluoride

As shown in the figure 12, with the increase of temperature and volumetric flow rate in dehydration of ethanol, the total flux increases. With the increase of temperature, the driving force of mass transfer and the saturated vapor pressure of useful compounds while penetration in membrane increases, and the flux increases accordingly.

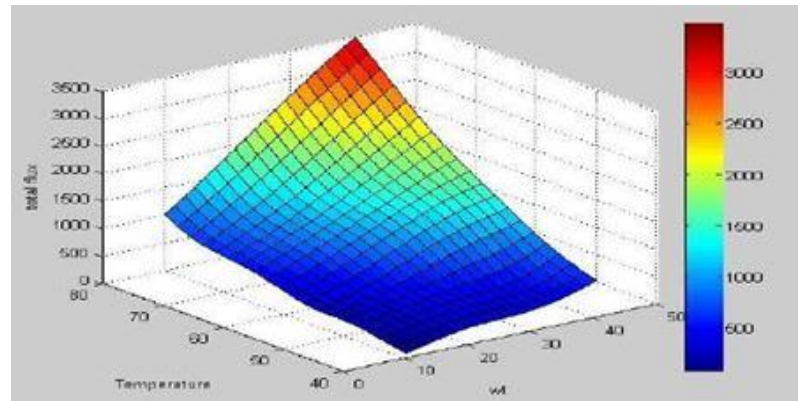

Figure.12 Figure graph for water- ethanol dehydration by ethanol Polydimethylsiloxane polymer membrane and Polyvinylidene fluoride

\section{3. modeling dehydration of organic compounds by use of COMSOL Multiphysics Software}

The organic compounds including acetone, butanol, ethanol, isopropanol, and methanol were utilized in this study, and the COMSOL Multiphysics version 4.2.0.150 was implemented to data analysis. The procedure description is as follows:

Mesh is the starting point for the Finite Element method that partitions geometry into simpler and smaller units.

In this method, you can see counters in the results section that includes temperature (ht), mass fraction, flux (chcs), velocity (spf), and pressure (spf).

Under the temperature of 3133.15 kelvin, the separation coefficient of 57 , the flux permeability of $1.023 \mathrm{~kg} / \mathrm{m} 2 \mathrm{~h}$ for dehydration of acetone aqueous solution of $20 \% \mathrm{wt}$, the pressure was equal to 70 pascal, and the membrane used was acrylonitrile and 2- Hydroxyethyl methacrylate grafted polyvinyl alcohol [12].

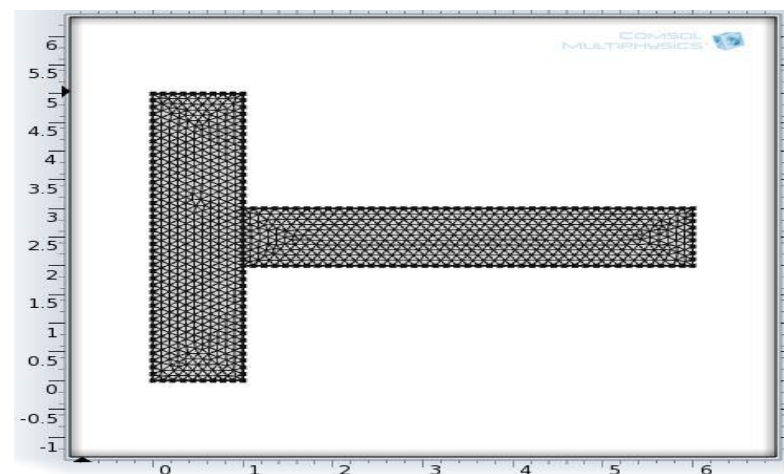

Figure.13 Meshing the membrane module in the wateralcohol processing by different membranes

In the temperature graph, the input temperature equals with the atmosphere temperature, and it gradually increases across the membrane, as condensation takes place in the membrane output due to the vacuum condition. Afterward, the temperature decreases thermodynamically in the membrane output, and becomes cold.

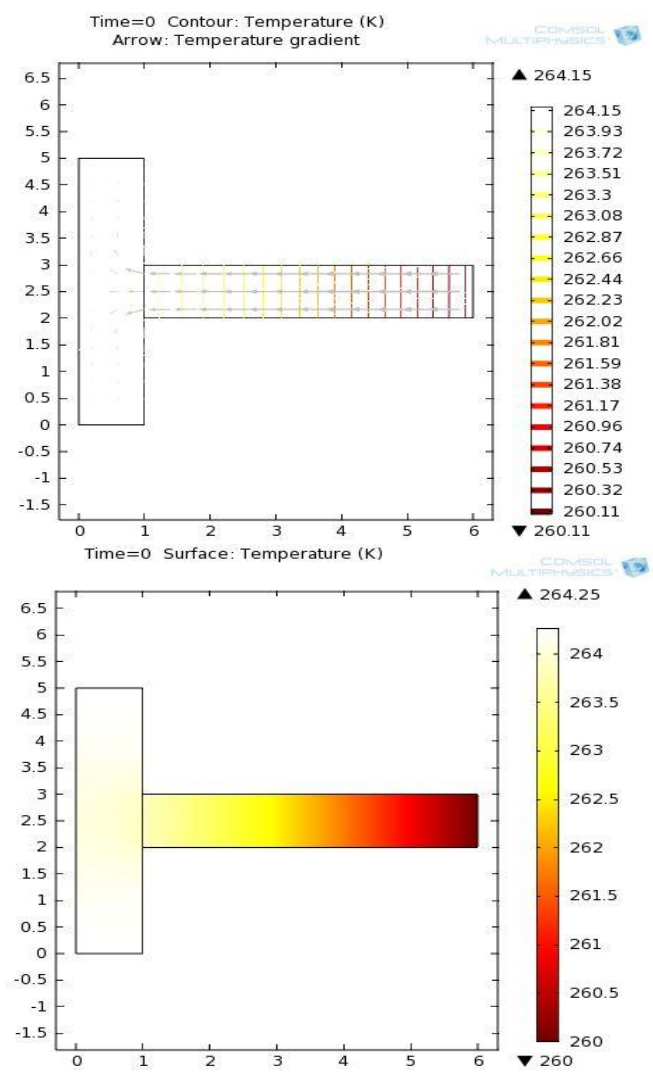

Figure.14 Temperature in water-acetone processing by Acrylonitrile and 2- Hydroxyethyl methacrylate grafted polyvinyl alcohol

As can be seen in the mass fraction graph 15, the amount of mass fraction is fixed across the module. The reason is that

\section{Mansoor Kazemimoghadam \& Zahra Amiri}


after balancing the membrane swelling and saturation concentration of water on the membrane surface, flux and water separation factor change slightly with the increase of the feed's water amount.

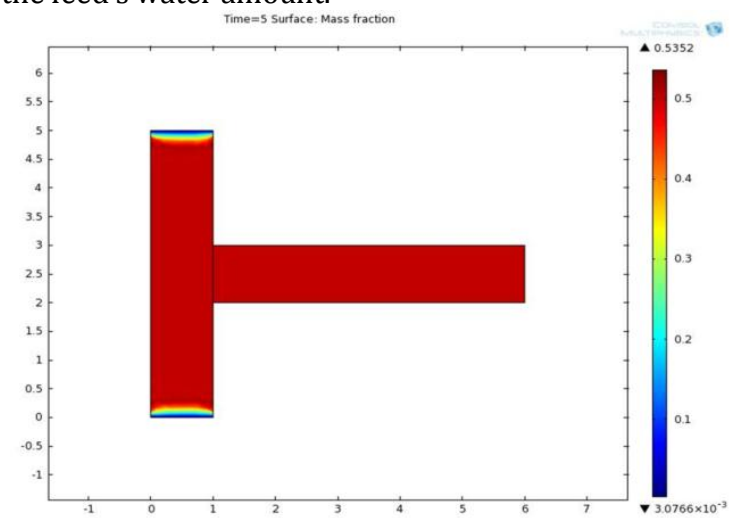

Figure.15 Mass fraction in water-acetone processing by Acrylonitrile and 2-Hydroxyethyl methacrylate grafted polyvinyl alcohol

As shown in the figure, flux is stable and low in the membrane entries and walls due to the low driving force. However, the flux increased within the membrane, as the pressure increased. The error percentage in flux was 3.049.

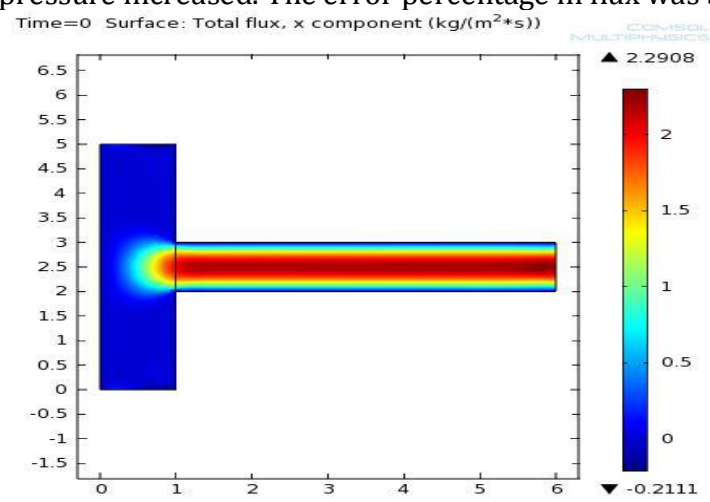

Figure.16 Flux graph for water-acetone processing by Acrylonitrile and 2-Hydroxyethyl methacrylate grafted polyvinyl alcohol

The current velocity in the entry is low, and it decreased across the walls too in the velocity graph; however, the velocity increased in the membrane. The reason of this phenomenon can be reduction of temperature across the membrane and increase of water concentration in membrane. Afterward, the separation factor for water increase due to the increase of the driving force for the mass transfer.

As observed in the figure, the input is under the atmosphere pressure, and the pressure decreased across the membrane module. The reason can be said to be reduction of the driving force across the membrane.

The error percentage for flux in dehydration of butanol, ethanol, isopropanol, and methanol were as follows:

Under the temperature of 333.15 , separation coefficient 26.5 , and permeability flow of $3.07 \mathrm{~kg} / \mathrm{m} 2 \mathrm{~h}$ for dehydration of butanol, 27.6\%wt was achieved. The pressure was 33.33 pascal and the membrane utilized was a combination of polyvinyl alcohol and nylon 66 [13]. The error percentage in flux was 3.7.

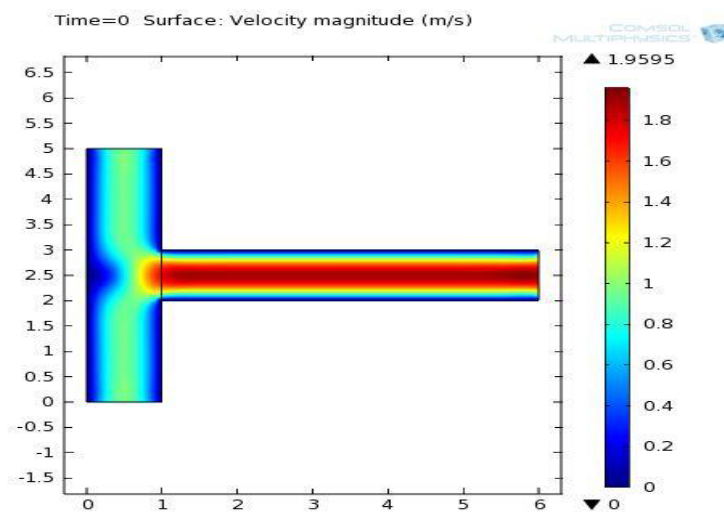

Figure.17 Velocity graph for water- acetone processing by Acrylonitrile and 2- Hydroxyethyl methacrylate grafted polyvinyl alcohol

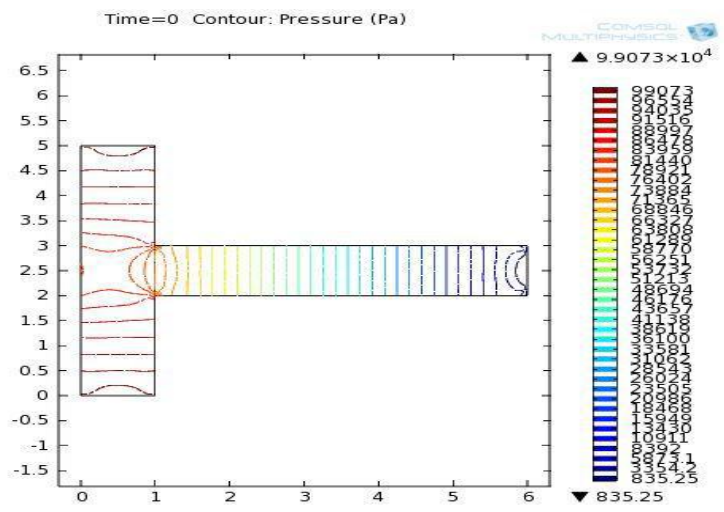

Figure.18 Pressure graph for water-acetone processing by Acrylonitrile and 2- Hydroxyethyl methacrylate grafted polyvinyl alcohol

Under the temperature of 333.15, separation coefficient 43, and permeability flow of $0.2 \mathrm{~kg} / \mathrm{m} 2 \mathrm{~h}$ for dehydration of ethanol, 95\%wt was achieved. The pressure was 40 pascal and the membrane utilized was Polyvinyl alcohol [14]. The error percentage in flux was 3.51.

Under the temperature of 343.15 , separation coefficient 1002 , and permeability flow of $1.35 \mathrm{~kg} / \mathrm{m} 2 \mathrm{~h}$ for dehydration of isopropanol, 50\%wt was achieved. The pressure was 180 pascal and the membrane utilized was the combination of polyvinyl alcohol and poly-electrolyte [15]. The error percentage in flux was 2.88 .

The temperature of 303.15 , separation coefficient 13 , and permeability flow of $1.8 \mathrm{e}-5 \mathrm{~kg} / \mathrm{m} 2 \mathrm{~h}$ for dehydration of methanol, 10\%wt was achieved. The pressure was 130 pascal and the membrane utilized was Poly-3hydroxybutyrate [16]. The error percentage in flux was 3.82 .

\section{Mansoor Kazemimoghadam \& Zahra Amiri}




\section{Comparison of Ann and Comsol Indehydration of Acetone by Polyacrylonitrile and Polyethylene Glycol}

The error value in the ANN was 1.86, and the error value in COMSOL was 2.11. Regarding the error value, it can be concluded that both modeling methods were appropriate, and the error percentage in ANN is lower than the COMSOL. As a result, ANN is more accurate, and the reason is that ANN considers the problems in detail, while COMSOL considered the problems in general.

\section{CONCLUSION}

In this study, dehydration of water-ethanol, water- aceton, and water butanol by use of pervaporation process was modeled in ANN. The polymer membranes Polydimethylsiloxane, Polyvinylidene fluoride, Polyacrylonitrile, and Polyethylene glycol are hydrophilic membranes, and are appropriate for separation of low amounts of water in alcohols. Moreover, the ANN in this study reflected the error suitably.

The dehydration of water-acetone, water-butanol, waterethanol, water-isopropanol, and water-mathanol by pervaporation process were also modeled in the COMSOL. The hydrophilic membranes were used that are good for separation of low amounts of water in alcohols. Moreover, the COMSOL in this study reflected the error suitably.

\section{REFERENCES}

[1] Henely,S., 1998. Separation process principles, John Wiley and Sons.

[2] Srikanth, G., 2008. Membrane separation processes technology and business, Opportunities. Water conditioning and purification.

[3] Baker, R., 2004. Membrane technology and application. John Wiley \& Sons, Ltd.

[4] Kujawski. W., 2000. Application of pervaporation and vapor permeation in environmental protection. Polish Journal of environmental studies, 9 (1), 13-26.

[5] Huang, R., 1001. Pervaporation membrane separation process. ELSEVIER, 11(120), 181-191.

[6] Feng, X., Huang, R., 2004. Liquid separation by membrane pervaporation A review, Industrial Baker. Membrane technology and application.

[7] Mulder, M., 1996. Basic principles of membrane technology. ed. S. Edition, Kluwer.

[8] Rautenbach, R., Albrecht, R., 1985. The separation potential of pervaporation: part 1. Discussion of transport equations and comparison with reserve osmosis. Journal of membrane science, 1 (23).

[9] Xia, Z., Ji-ding, L., Jian, C., Jun-qi, H., 2009. Pervaporation of ethanol/water mixtures with high flux by zeolite-filled PDMS/PVDF composite membranes. Chinese Journal of polymer science, 771780.

[10] Qiang, Z., Jinwen, Q., Quanfu, A., Zhihui, Z., Peng, Z., Yunxiang, B., 2008. Studies on pervaporation characteristics of polyacrylonitrile-b-poly (ethylene glycol)-b polyacrylonitrile block copolymer membrane for dehydration of aqueous acetone solutions. Journal of membrane science, 311, 284-293.
[11] Elsayed, A., Xianshe, F., 2009. Pervaporation separation of n-butanol from dilute a aqueous solutions using silicalite-filled poly (dimethyl siloxane) membranes. Journal of Membrane Science, 339, 120-125.

[12] Merve, 0., 2015. A novel in situ synthesized magnetite containing acrylonitrile and 2-hydroxyethylmethacrylate grafted poly (vinyl alcohol) nanocomposite membranes for pervaporation separation of acetone/water mixtures. Journal of Membrane Science, 1-10.

[13] Sridhar, S., Smitha, B., Amarnath, A., 2006. Separation of 2-butanol-water mixtures by pervaporation through PVA-NYL 66 blend membranes. Membrane Separations Group, Chemical Engineering Division, Indian Institude of chemical technology.

[14] Takegami, S., Yamada, H., Tsujii, S., 1992. Dehydration of water/ethanol mixtures by pervaporation using modified poly(vinyl alcohol) membrane. Toyobo Research Institute.

[15] Zhao, Q., Qian, J., An, Q., Meihua, z., 2009. Poly(vinyl alcohol)/polyelectrolyte complex blend membrane for pervaporation dehydration of isopropanol. Department of polymer science and engineering.

[16] Mercedes, V., Vidaurre, C., 2014. Gottifredi, Sorption and pervaporation of methanol/water mixtures with poly (3hydroxybutyrate) membranes.

Mansoor Kazemimoghadam \& Zahra Amiri 